\title{
Effect of Oligomerization of Counterions on Water Activity in Aqueous Cationic Surfactant Systems
}

\author{
Vitaly Kocherbitov ${ }^{1,2 *}$ and Olle Söderman ${ }^{3}$ \\ ${ }^{1}$ Biomedical Science, Faculty of Health and Society, Malmö University, SE-20506 Malmö, Sweden \\ ${ }^{2}$ Biofilms Research Center for Biointerfaces, Malmö University, SE-20506 Malmö, Sweden \\ ${ }^{3}$ Physical Chemistry, Center for Chemistry and Chemical Engineering, P.O. Box 124, Lund University, \\ SE-22100 Lund, Sweden
}

Supporting information 
Sorption isotherms presented as functions of water activity
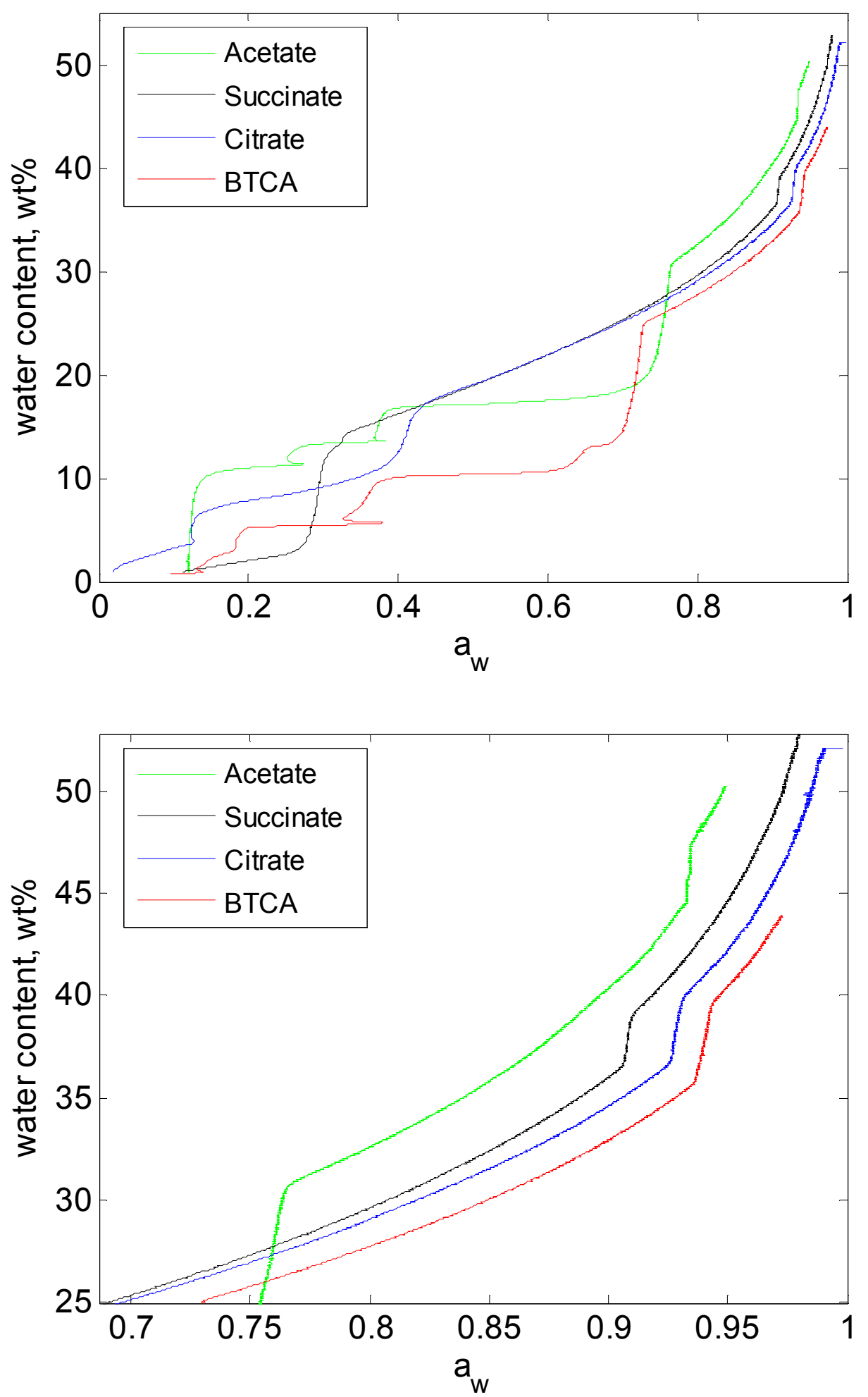

Figure S1. Water content as function of water activity for the four surfactants 
Calculation of the volume fraction of rods in the hexagonal phase

The volume fraction of rods $\Phi_{\text {rod }}$ was calculated as the volume fraction of CTA- ions:

$$
\Phi_{\text {rod }}=\frac{V_{C T A}}{V_{w}+V_{c i}+V_{C T A}}=\frac{m_{C T A} / d_{C T A}}{m_{w} / d_{w}+m_{c i} / d_{c i}+m_{C T A} / d_{C T A}}=\frac{\frac{x_{S} M_{C T A} n_{C T A}}{M_{S} d_{C T A}}}{x_{w} / d_{w}+\frac{x_{S} M_{c i}}{M_{s} d_{c i}}+\frac{x_{S} M_{C T A} n_{C T A}}{M_{S} d_{C T A}}}
$$

Using the group contribution method (Hagslätt, H.; Söderman, O.; Jönsson, B. Liq. Cryst. 1992, 12, 667-688), the density value $d_{C T A}=0.8365$ was obtained and used in the volume fraction calculation (the group volumes are shown in Table S1). The densities of counterions obtained by the same method are listed in Table S2.

Table S1. Group volumes at $25^{\circ} \mathrm{C}$ used in calculations of densities

\begin{tabular}{|l|l|}
\hline Group & Volume, $\AA^{3}$ \\
\hline $\mathrm{CH}_{3}$ & 54.19 \\
\hline $\mathrm{CH}_{2}$ & 26.99 \\
\hline $\mathrm{CH}$ & $26.99 *$ \\
\hline $\mathrm{C}$ (in citrate, from vdW radius) & 20.6 \\
\hline $\mathrm{COO}-$ & 35.0 \\
\hline $\mathrm{N}\left(\mathrm{CH}_{3}\right)_{3}$ & 105.8 \\
\hline
\end{tabular}

* The volume of $\mathrm{CH}$ is approximated as the volume of $\mathrm{CH}_{2}$

Table S2. Molecular masses and densities used in calculations of volume fractions. $M_{s}$ stands for the total molar mass of surfactant. The molecular mass of the cationic part $M_{C T A}=284.55$

\begin{tabular}{|l|c|c|l|c|}
\hline Counterion & $M_{c i}$ & $M_{S}$ & $M_{c i} / M_{S}$ & $d_{c i}$ \\
\hline Ac $^{-}$ & 59.04 & 343.6 & 0.1718 & 1.0993 \\
\hline Succ $^{2-}$ & 116.1 & 685.2 & 0.1694 & 1.5547 \\
\hline Citr $^{3-}$ & 189.1 & 1042.7 & 0.1814 & 1.5974 \\
\hline BTC $^{4-}$ & 230.1 & 1368.3 & 0.1682 & 1.5412 \\
\hline
\end{tabular}

\section{Calculation of the volume fraction of counterions}

The volume fraction of counterions in the water layer $\varphi_{c i}$ can be calculated from volumes of counterions and water:

$$
\varphi_{c i}=\frac{V_{c i}}{V_{c i}+V_{w}}=\frac{1}{\frac{V_{w}}{V_{c i}}+1}=\frac{1}{\frac{m_{w} d_{c i}}{d_{w} m_{c i}}+1}
$$

The mass of counterions $m_{c i}$ can be calculated as follows:

$$
m_{c i}=m_{s} \frac{M_{c i}}{M_{s}}
$$

where $M_{S}$ is the total molecular mass of the surfactant, $M_{c i}$ is the molecular mass of the counterion. Then the volume fraction of counterions can then be calculated knowing the water to surfactant mass ratio:

$$
\varphi_{c i}=\frac{1}{\frac{m_{w} M_{s} d_{c i}}{m_{s} M_{c i} d_{w}}+1}
$$

The parameters used in the calculation are shown in Table S2. 
Solid-state behavior of the four surfactants

Acetate:
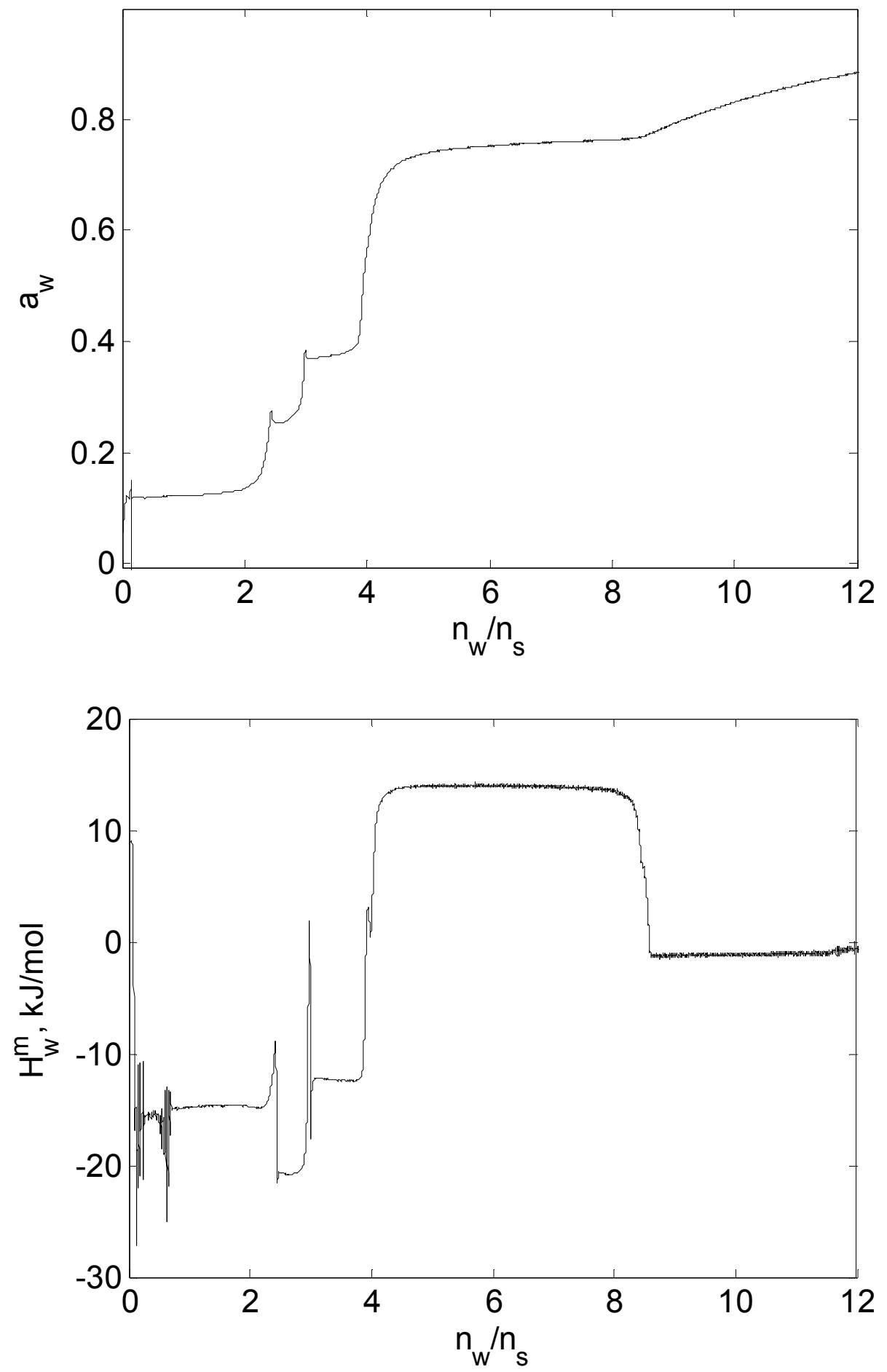

Figure S2. The activity of water and the partial molar enthalpy of mixing of water as functions of water to CTA acetate molar ratio at low water contents 
Succinate:
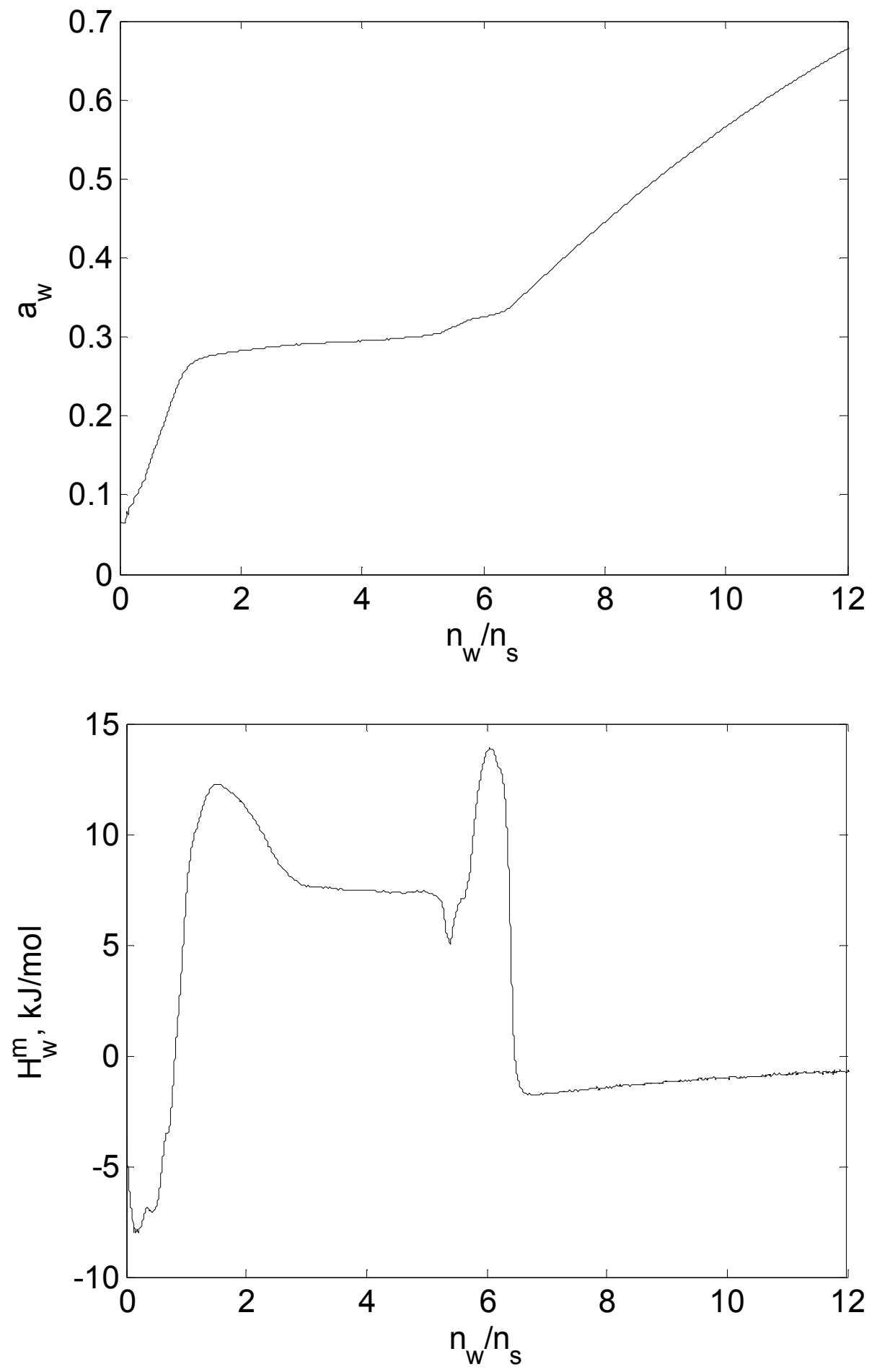

Figure S3. The activity of water and the partial molar enthalpy of mixing of water as functions of water to CTA succinate molar ratio at low water contents 


\section{Citrate:}

The blue and the black curves show results of two different experiments
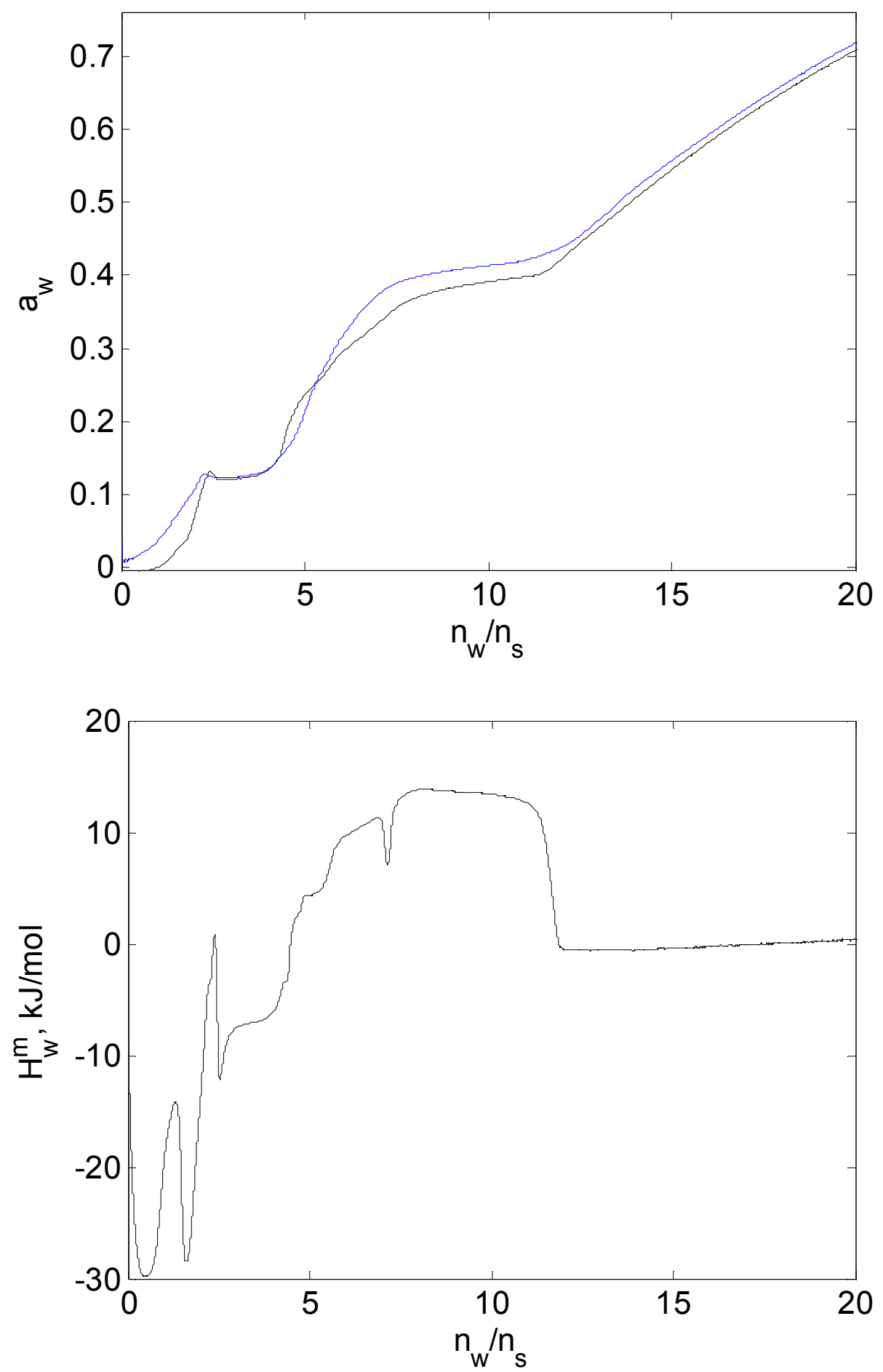

Figure S4. The activity of water and the partial molar enthalpy of mixing of water as functions of water to CTA citrate molar ratio at low water contents 
BTCA:
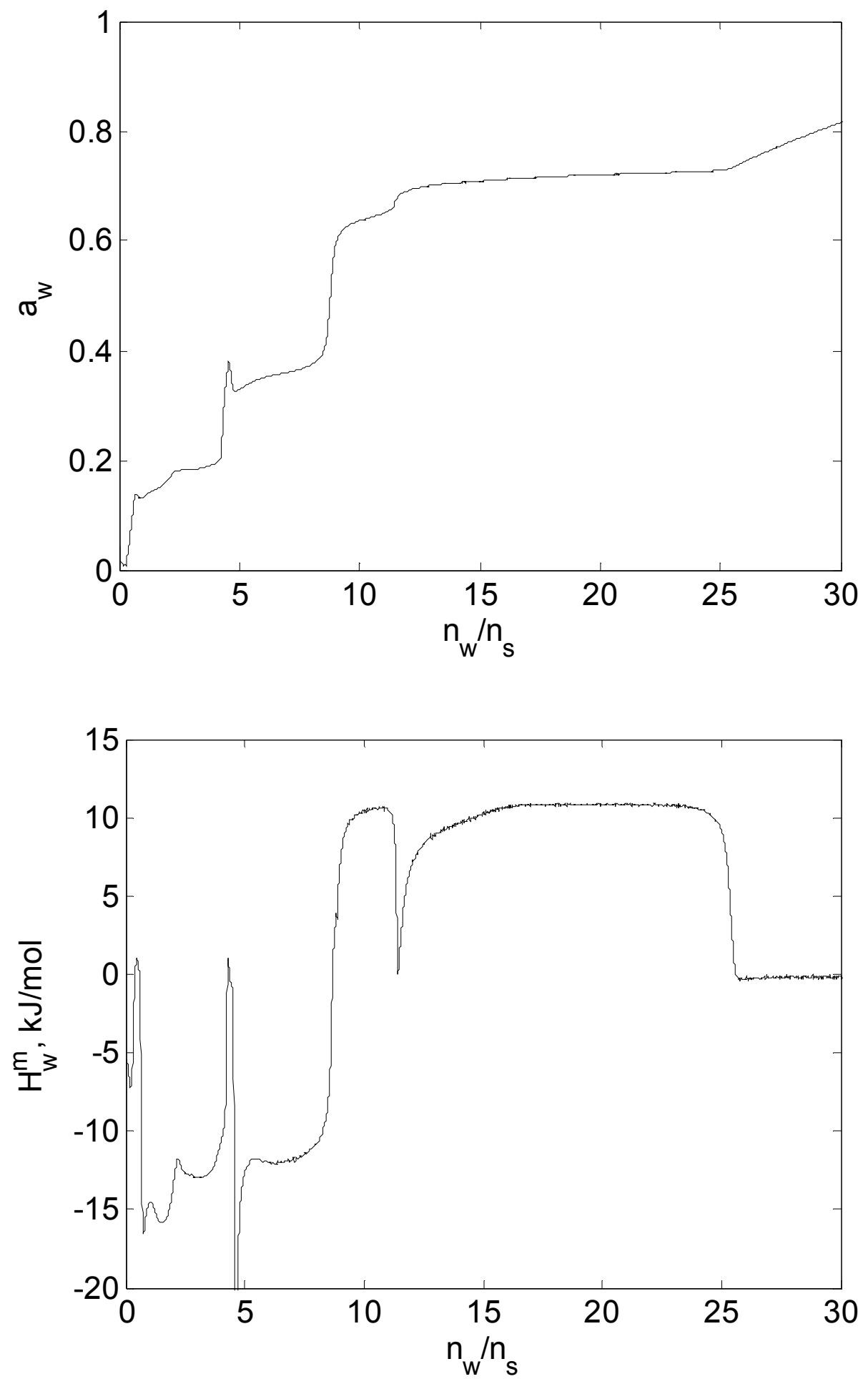

Figure S5. The activity of water and the partial molar enthalpy of mixing of water as functions of water to CTA BTCA molar ratio at low water contents 
The meaning of the enthalpy parameter in the van der Waals differential equation

The van der Waals differential equation is a generalization of Clapeyron equation for binary systems:

$$
\left(\frac{d T}{d x}\right)^{(1)}=-\frac{R T^{2}\left(\frac{d \ln a_{w}}{(1-x) d x}\right)_{T, P}^{(1)} \Delta x^{1 \rightarrow 2}}{H^{1 \rightarrow 2}}
$$

where $a_{w}$ is the activity of water and $x$ is the mole fraction (the equation is general and can be used also for non-aqueous binary systems). This equation allows calculation of the slopes of phase boundaries $d T / d x$ at constant pressures. The enthalpy parameter $H^{1 \rightarrow 2}$ needed for these calculations is defined below:

$$
H^{1 \rightarrow 2}=H^{(2)}-H^{(1)}-\Delta x^{1 \rightarrow 2}\left(\frac{\partial H}{\partial x}\right)_{T, P}^{(1)}
$$

This form of the enthalpy parameter follows from the fact that in binary systems the phases in equilibrium have different compositions (in the present case different water contents $x$ ).

In the sorption calorimetric experiments, the measured enthalpy parameter is the partial molar enthalpy of mixing of water $H_{w}^{m}$. This is a derivative of enthalpy of mixing, therefore to calculate $H^{1 \rightarrow 2}$ one needs to integrate $H_{w}^{m}$ :

$$
H^{1 \rightarrow 2}=\left(1-x_{w}^{(2)}\right) \int_{r^{(1)}}^{r^{(2)}}\left(H_{w}^{m}-H_{w}^{m(1)}\right) d r
$$

Schematic curves of enthalpy $H$ and of the partial molar enthalpy of mixing of water $H_{w}^{m}$ close to the phase transition are shown in Figure S6 (next page). The dashed lines show the course of the curves in a hypothetical (metastable) situation when a phase transition does not happen. When integrating $H_{w}^{m}$, this curve (line) serves as a baseline for integration. More details can be found in the following references:

van der Waals, J. D.; Kohnstamm, P., Lehrbuch der Thermostatik : das heisst des thermischen Gleichgewichtes materieller Systeme. Johann Ambrosius Barth: Leipzig, 1927 Kocherbitov, V., Driving forces of phase transitions in surfactant and lipid systems. J. Phys. Chem.B. 2005, 109 (13), 6430-5.

Kocherbitov, V., Application of scanning methods to distinguish between entropy and enthalpy driven phase transitions. Curr. Opin. Colloid Interface Sci. 2013, 18, 510-516.

Kocherbitov, V., Salt-saturated salt solution as a standard system for sorption calorimetry. Thermochim. Acta 2004, 421, 105-110

Kocherbitov, V.; Söderman, O.; Wadsö, L., Phase Diagram and Thermodynamics of the n-Octyl - D Glucoside / Water System. J. Phys. Chem. B 2002, 2910-2917 

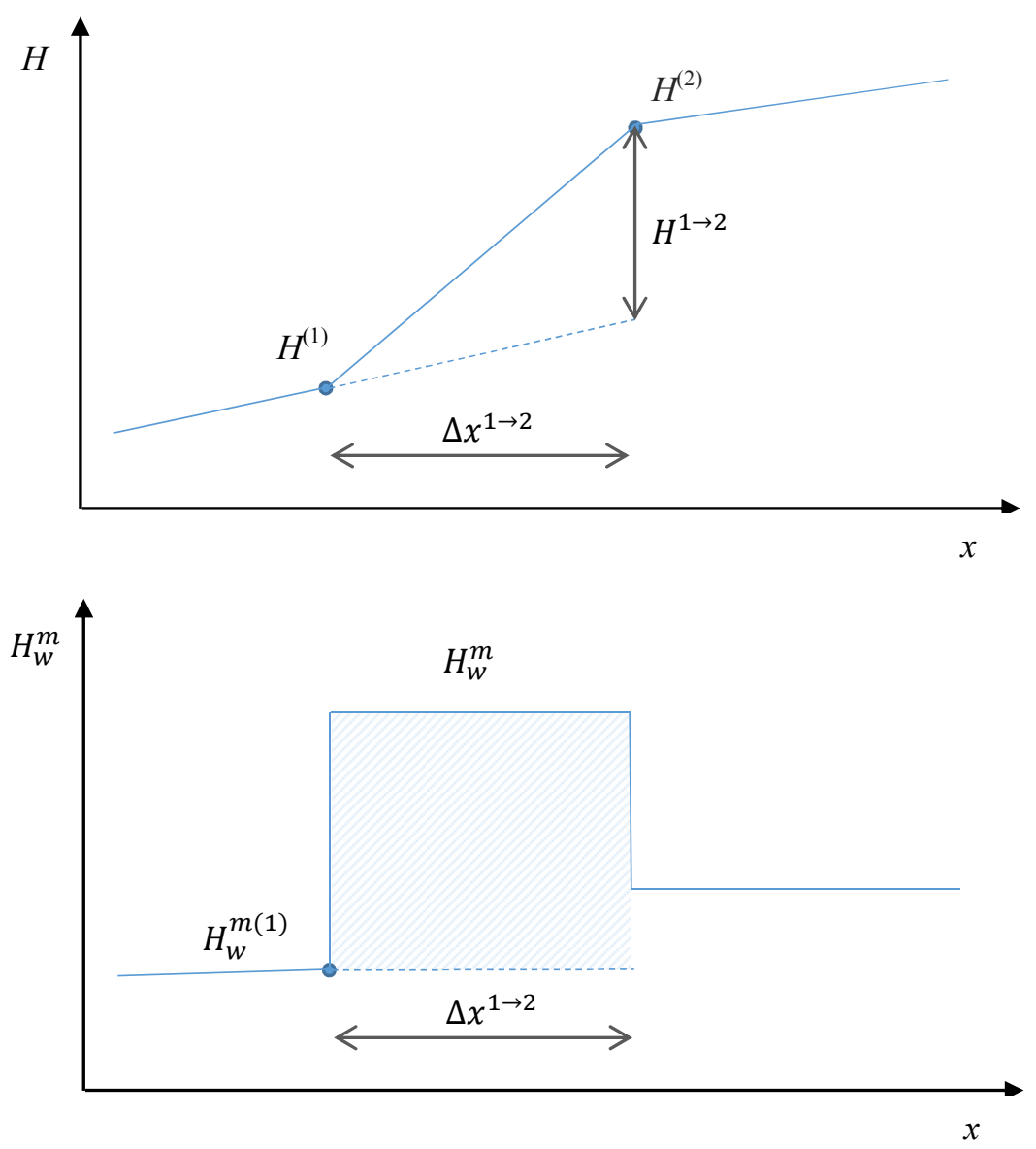

Figure S6. A schematic illustration of enthalpy curves in the vicinity of an isothermal phase transition from phase 1 to phase 2 . 